\title{
The “Texas Two-Step” procedure
}

\author{
Edward Y. Chan, MD, ${ }^{\mathrm{a}}$ Ross M. Reul, MD, ${ }^{\mathrm{b}}$ Min P. Kim, MD, ${ }^{\mathrm{a}}$ and Michael J. Reardon, MD, ${ }^{\mathrm{b}}$ Houston, Tex
}

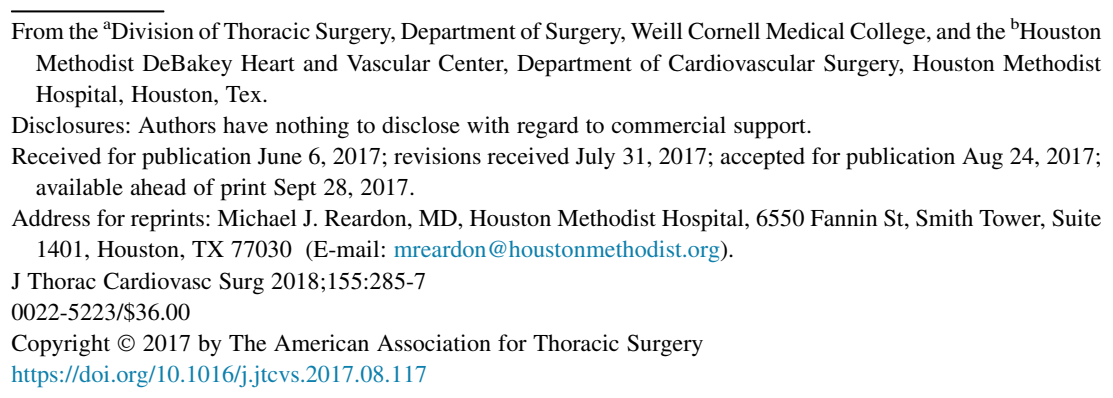

As a referral center with a multidisciplinary cardiac tumor program, we evaluate a high volume of primary cardiac sarcomas and other complex cardiac tumors, occasionally requiring both cardiac resection and pneumonectomy to achieve complete resection. Our previous experience identified as at prohibitively high risk those patients who undergo extensive cardiac resection with concomitant pneumonectomy, with a 30-day surgical mortality of $43 \%$. ${ }^{1}$ These patients have dense pleural adhesions requiring adhesiolysis during the pneumonectomy. Post-cardiopulmonary bypass coagulopathy has resulted in postoperative bleeding into the pneumonectomy space, leading to transfusions, unilateral pulmonary edema, and death.

\section{MATERIALS AND METHODS}

For our last 3 consecutive patients who required extensive cardiac resection and pneumonectomy, we developed a staged strategy of cardiac resection and reconstruction on cardiopulmonary bypass through a median sternotomy, with delayed pneumonectomy through a posterolateral thoracotomy: the "Texas Two-Step." The study was performed with institutional review board approval and individual patient consent.

\section{CLINICAL SUMMARIES}

\section{Patient 1}

For a 38-year-old man with a pulmonary artery (PA) angiosarcoma, we resected his pulmonary root and both main PAs to the first bifurcation. The left PA was completely occluded with tumor and was divided sharply, with no back bleeding. A small amount of tumor was removed with an endarterectomy, and the adventitia was closed. The right PA was partially occluded with tumor, so a PA tumor endarterectomy was performed. We reconstructed the PA root with a $25-\mathrm{mm}$ pulmonary allograft and used an 18-mm Dacron polyester fabric graft for the right main PA. The divided left PA was left in place. (Figure 1). Our cannulation and cardioplegia strategy was consistent with our earlier experience and has been previously detailed. ${ }^{1}$ On postoperative day (POD) 3, left pneumonectomy was performed. The patient was discharged home on POD 18 . Final pathologic examination demonstrated a high-grade last 9 months.

\section{Patient 2} last 9 months.

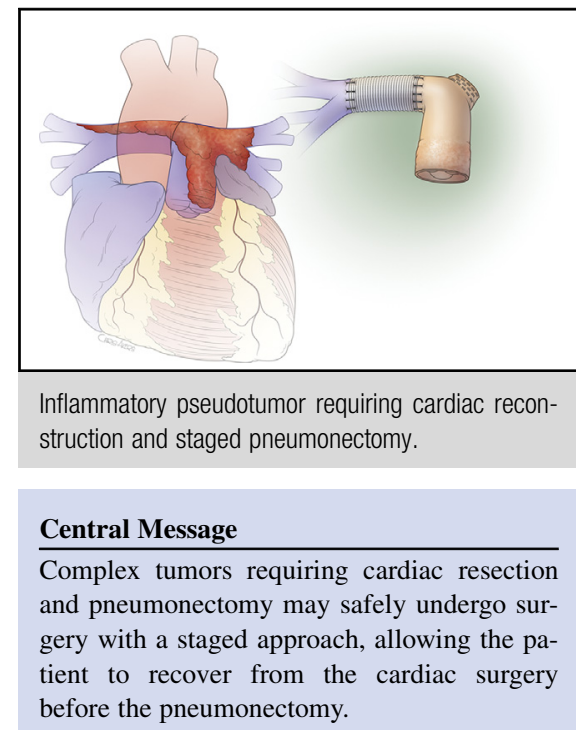

See Editorial Commentary page 288.

3.5-cm pleomorphic sarcoma of the PA with negative lymph nodes. He has been seen regularly in follow-up during the

A 40-year-old woman with a right lung inflammatory pseudotumor invading her heart was referred to our program for resection. Multiple medical therapies had failed, and she continued to have severe symptoms with chest pain. The patient underwent cardiac resection of the inflammatory pseudotumor en bloc with the superior vena cava, right atrium, dome of the left atrium, right pulmonary veins, and right main PA, followed by reconstruction with bovine pericardium (Figure 2). The inflammatory process of her primary disease had effectively destroyed her right lung and resulted in dense adhesions to the adjacent cardiac structures. Because of complications after the cardiac resection, her pneumonectomy was delayed until POD 16, when she underwent a right pneumonectomy. She was discharged to a long-term acute care hospital on POD 39. Final pathologic examination demonstrated a $9.5-\mathrm{cm}$ inflammatory pseudotumor with negative mediastinal lymph nodes. A delayed hemothorax developed while she was an outpatient on anticoagulation with empyema and bronchopleural fistula. She underwent an Eloesser flap with omental flap, after which she returned to long-term acute care. She has been followed up regularly during the 


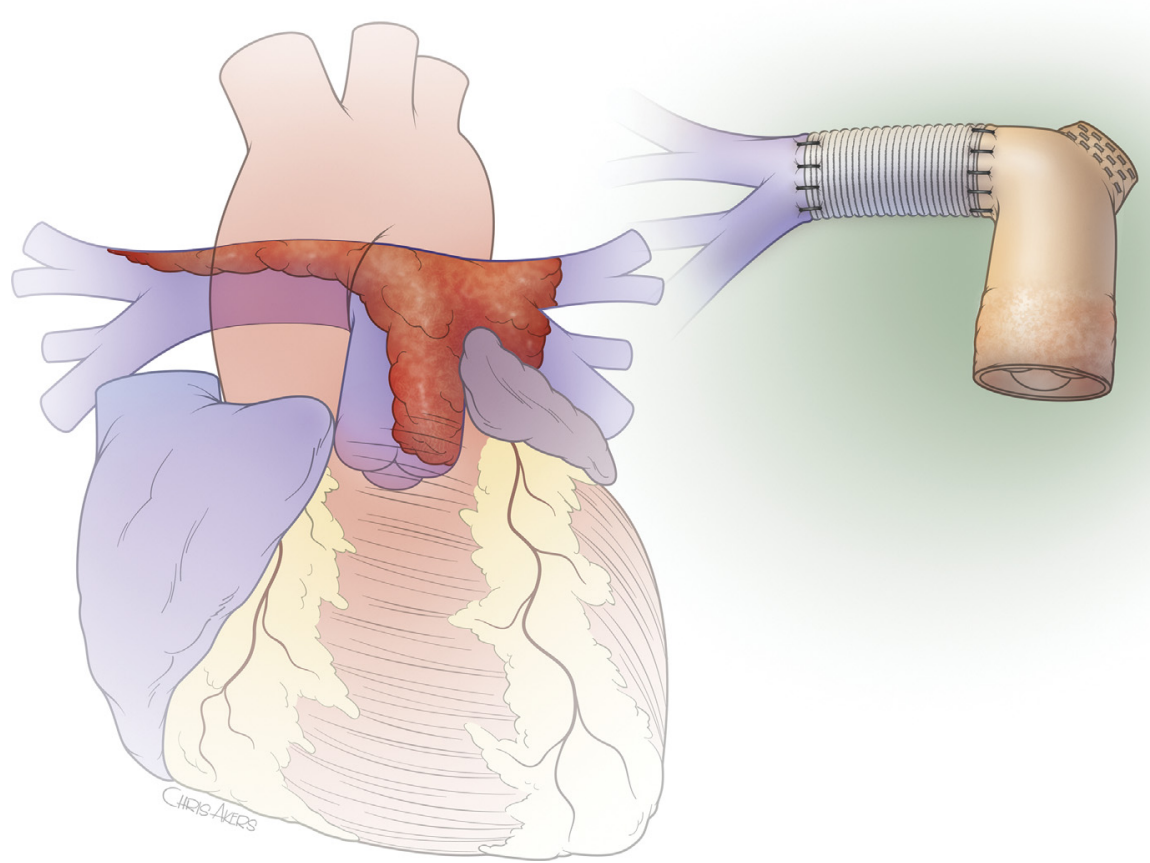

FIGURE 1. Pulmonary artery angiosarcoma extending distally into the right pulmonary artery and completely occluding the left pulmonary artery. The tumor was resected and reconstructed with a pulmonary allograft for the main pulmonary artery and a Dacron polyester fabric graft for the right pulmonary artery.

\section{Patient 3}

The third patient is a 27-year-old man with a large right lung synovial sarcoma also involving his heart. He underwent left atrial resection and reconstruction with bovine pericardium and proximal right PA division. On POD 1, he underwent right pneumonectomy. Dense

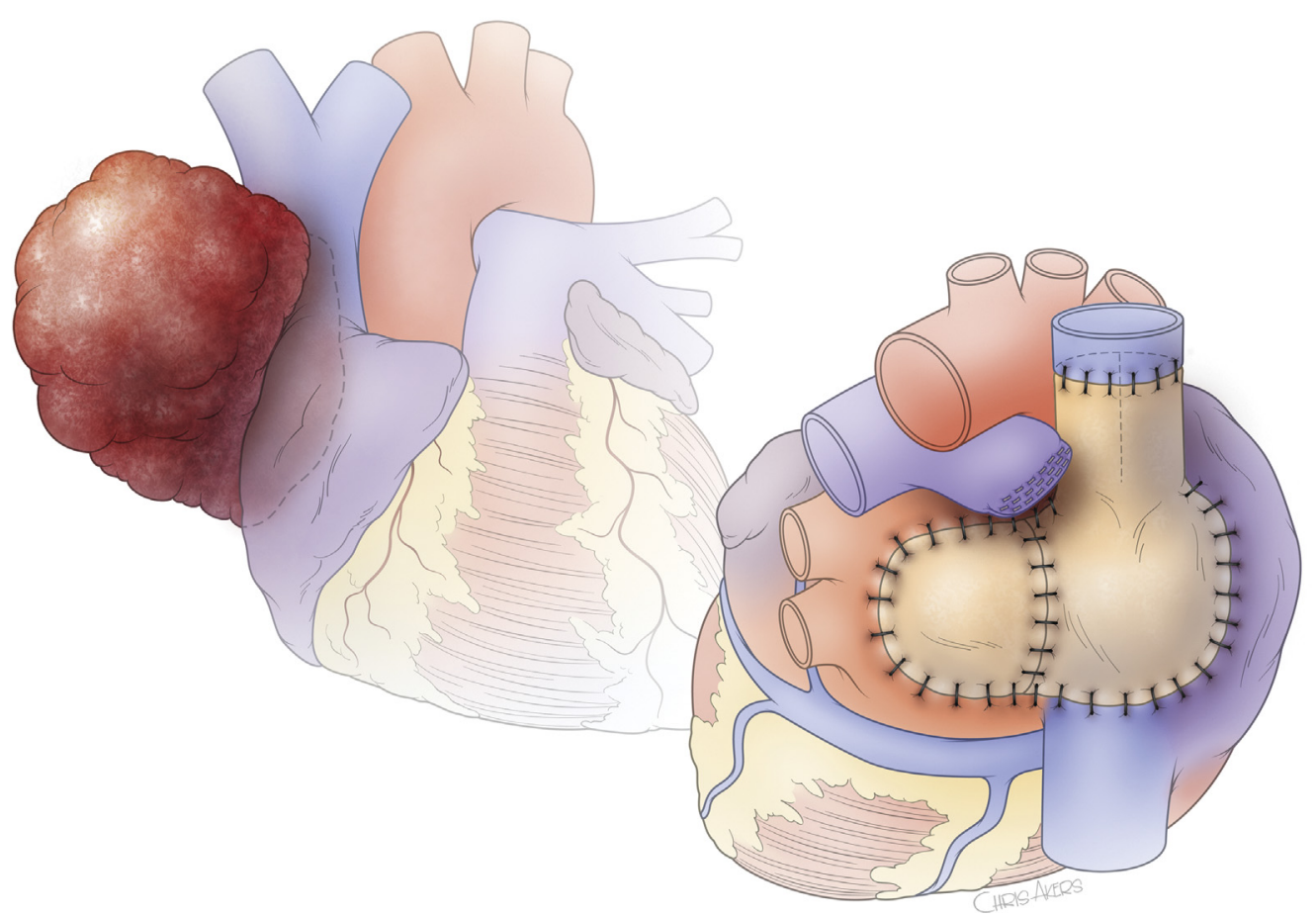

FIGURE 2. Inflammatory pseudotumor originating in the right lung and invading the adjacent structures. The tumor was resected and reconstructed with a bovine pericardial patch for the left atrium and a tubularized bovine pericardial patch for the superior vena cava and right atrium. 
adhesions were encountered, which required extensive adhesiolysis. His postoperative course was complicated only by atrial fibrillation, and he was discharged home on POD 13. Final pathologic examination demonstrated a $15-\mathrm{cm}$ stage III synovial sarcoma with negative lymph nodes and a positive margin only at the inferior vena cava, for which we recommended stereotactic body radiation therapy. He has been seen in clinic for his 4-month follow-up.

\section{DISCUSSION}

Malignant and complex tumors that require both extensive cardiac resection on cardiopulmonary bypass and pneumonectomy can present a challenge to the cardiothoracic surgeon, especially when significant chest wall adhesions exist. Leaving the lung in place after cardiac resection avoids a pneumonectomy space during the immediate postoperative period, when coagulopathy can be problematic. In determining the timing for pneumonectomy, we wait at least 1 day after the cardiac resection to allow correction of coagulopathy and metabolic derangements and then use mediastinal tube output, hemodynamic stability, and respiratory status as indicators to proceed. Our strategy has resulted in substantially lower transfusion requirements, and no patients have had recurrent disease to this point. Generally, these patients do not require adjunctive methods of postoperative respiratory support, such as extracorporeal membrane oxygenation, because they have effectively been living with single-lung physiology leading up to surgery. Although this report represents an early stage in our experience, we believe that this staged technique is a useful and potentially safer approach to this challenging population.

The authors wish to acknowledge Mr Chris Akers for his contribution in creating the illustrations for the images.

\section{Reference}

1. Ramlawi B, Al-Jabbari O, Blau LN, Davies MG, Bruckner BA, Blackmon SH, et al. Autotransplantation for the resection of complex left heart tumors. Ann Thorac Surg. 2014;98:863-8. 